
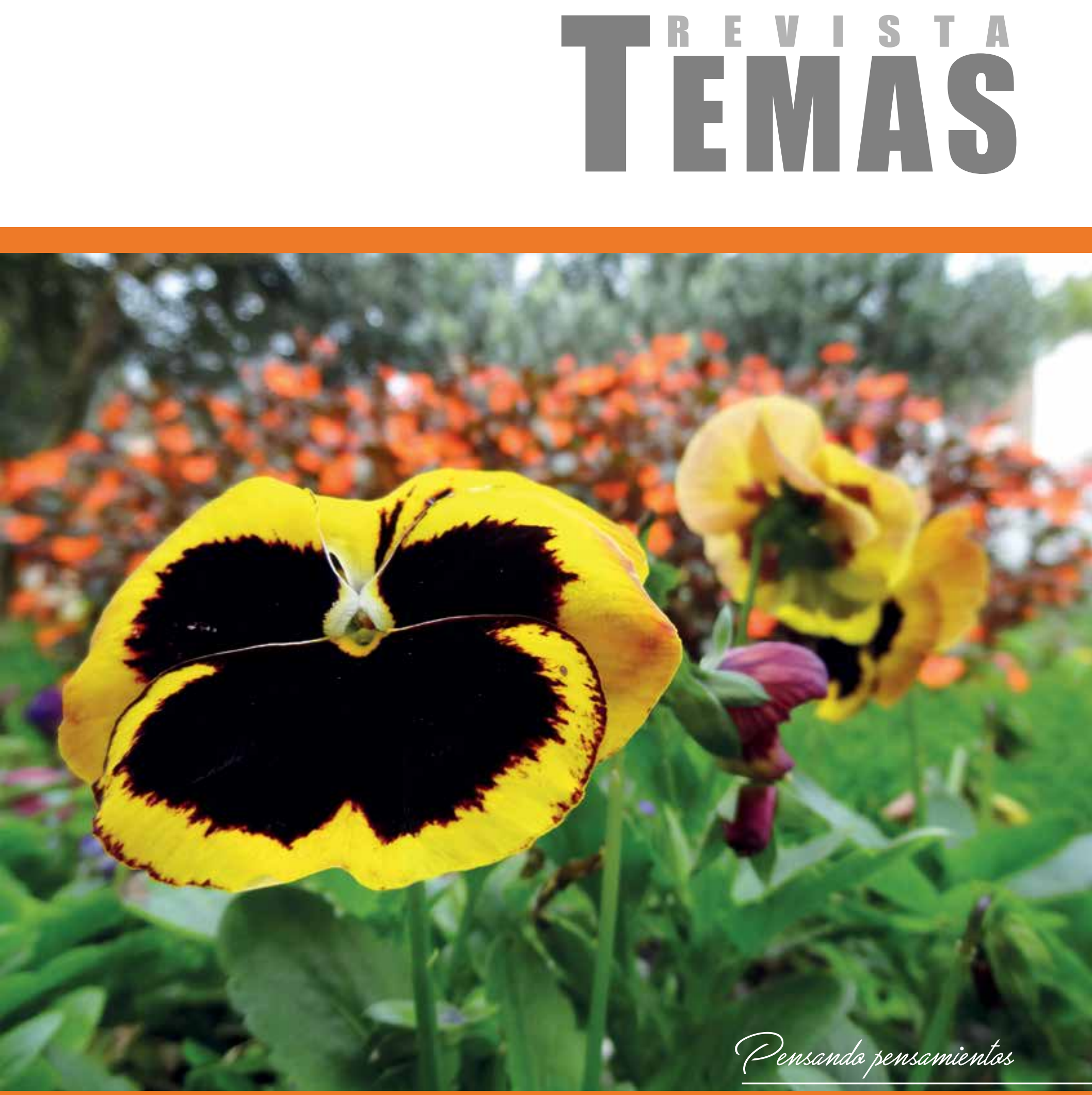

Referencia al citar este artículo:

Ossa, Y., y Quintero, M. (2016). Valoración de las competencias profesionales específicas en estudiantes de psicología de la Corporación Universitaria Minuto de Dios, sede Bello, que realizaron las prácticas profesionales durante el 2014. REVISTA TEMAS, 3(10), 123 - 133

\title{
Valoración de las competencias profesionales específicas en estudiantes de psicología de la Corporación Universitaria Minuto de Dios, sede Bello, que realizaron las prácticas profesionales durante el $2014^{1}$
}

\author{
Yomar de Jesús Ossa Henao² \\ Jessica Marcela Quintero Jurado ${ }^{3}$ \\ Recibido: 3/25/2016 Aceptado: 4/19/2016
}

\section{Resumen}

La presente investigación se propuso valorar las competencias profesionales específicas en estudiantes de psicología de la Corporación Universitaria Minuto de Dios, sede Bello, que realizaron las prácticas profesionales durante el 2014. Se hizo una revisión documental, teniendo como principal estrategia metodológica el análisis de contenido. Los materiales documentales fueron 259 proyectos e informes finales de prácticas, que se sistematizaron para construir las competencias por valorar. Inicialmente se sistematizaron cuatro variables: autores, técnicas, campo ocupacional y demandas. A partir de ellas se construyeron cuatro competencias: fundamentación teórica, búsqueda bibliográfica, recursos de intervención, e integración teórico-contextual. Se encontró que los estudiantes citan textos de diversas áreas del conocimiento, en especial de psicología y educación, siendo en su mayoría textos contemporáneos. El mayor porcentaje de proyectos referencian técnicas que no pertenecen al campo disciplinar de la psicología, incluyendo técnicas de carácter lúdico y artístico, resaltando su insuficiencia para responder a las demandas formuladas. Se identificó amplia variabilidad y diversidad en las demandas hechas a los estudiantes, algunas de ellas, como el consumo de sustancias psicoactivas, la educación sexual y la intervención familiar, fueron comunes a diferentes campos ocupacionales.

\section{Palabras clave}

Competencias profesionales, competencias específicas, valoración, prácticas profesionales, psicología.

\section{Assessment of the specific professional competencies of the Minuto de Dios psychology students -located in Bello-, who did the professional practices in 2014}

\section{Abstract}

This research aimed to assess the specific skills in psychology students at the University Corporation Minuto de Dios, located in Bello, who performed professional practices during 2014. A documentary review was conducted, with the main methodological strategy content analysis. The documentary materials were 259 projects and final reports of practices, which were systematized to build the skills to assess. authors, techniques, occupational field and demands: Initially four variables were systematized. Theoretical foundation, literature search, intervention resources, and theoretical and contextual integration: From these four races were built. It was found that students cite texts from different areas of knowledge in psychology and special education, being mostly contemporary texts. The highest percentage of projects referenced techniques that do not belong to the disciplinary field of psychology, including techniques playful and artistic character, highlighting its failure to respond to the demands. Wide variability and diversity in the demands that were made to students, some of them such as substance use, sex education and family intervention were common to different occupational fields identified.

\section{Keywords}

Professional Skills, specific skills, assessment, professional practices, psychology.

1 Artículo de Investigación.

2 Psicólogo y Especialista en Gerencia Educativa por la Universidad de San Buenaventura. Docente investigador de la Corporación Universitaria Minuto de Dios, sede Bello. Correo electrónico: yomarossahenao@gmail.com

3 Psicóloga por la Universidad de San Buenaventura. Magíster en Salud Mental de la Niñez y la Adolescencia por la Universidad CES. Docente investigadora de la Corporación Universitaria Minuto de Dios, sede Bello. Correo electrónico: jessicaquinteroj@gmail.com 


\section{Introducción}

A partir de la Ley 749 de 2002, el Ministerio de Educación Nacional asumió la tarea de mejorar la articulación entre los distintos niveles de la educación formal y de presentar referentes comunes que garanticen una educación de calidad en el país, independientemente de la región o naturaleza de la oferta educativa pública o privada. A partir de este momento, los proyectos educativos se articulan desde el concepto de competencia, como una manera de responder a las demandas del proceso de transformación social caracterizado por los avances tecnológicos, económicos y políticos, que han marcado los horizontes desde los cuales debe pensarse la educación. Como bien lo dice Climént (2014) la respuesta a estos cambios desde la educación, hace de las competencias "una sofisticada tecnología social con posibilidades de transferencia y desarrollo a gran escala" (p. 72). Se hace necesario entonces, clarificar el concepto de competencia para facilitar el consenso y la operacionalización del mismo, evitando así que se presente como un discurso pedagógico modernizante avalado sin ninguna rigurosidad teórica (Tobón, 2007).

El concepto de competencia se ha presentado como disperso, múltiple y polisémico. En respuesta a esta problemática, Tobón (2007) propone ubicarlo dentro del paradigma de la complejidad y la categoría del desarrollo humano para hacerlo más asible al momento de incluirlo en los diferentes proyectos educativos. El autor conceptualiza las competencias como:

procesos complejos que las personas ponen en acción-actuación-creación, para resolver problemas y realizar actividades (de la vida cotidiana y del contexto laboral-profesional), aportando a la construcción y transformación de la realidad, para lo cual integran el saber ser (automotivación, iniciativa y trabajo colaborativo con otros), saber conocer (observar, explicar, comprender y analizar) y el saber hacer (desempeño basado en procedimientos y estrategias) (p. 49).

Este concepto de competencias hunde sus raíces en el paradigma de la complejidad, que piensa el conocimiento, no como una reducción a una sola categoría, información, percepción, idea, descripción o teoría, sino como una actividad en la cual deben concebirse "diversos modos o niveles" (Morin, 1999, p. 20). A partir de este paradigma, la competencia adquiere un matiz que favorece la integración de productos, procesos y tendencias, mediante el surgimiento de una "nueva inteligencia y racionalidad que trascienda la parcelación y la fragmentación con el fin de que aborde la realidad en su multidimensionalidad" (Tobón, 2007, p. 46).

Tobón (2007) retoma la clasificación de las competencias de Juliao (1999) que consiste en dividirlas en básicas, genéricas y específicas. Las básicas se describen como fundamentales para vivir en sociedad y desenvolverse en cualquier ámbito laboral. Constituyen la base sobre la cual se forman los demás tipos de competencias. Las genéricas, por su parte, son comunes a varias ocupaciones u oficios y posibilitan la vinculación al entorno laboral y la movilidad entre diferentes empleos. Se adquieren mediante procesos sistemáticos de enseñanza y aprendizaje. Y por último, las específicas, son aquellas competencias propias de una determinada ocupación o profesión. Tienen un alto grado de especialización y, por tanto, especificidad en los procesos educativos. Este estudio se centró en la valoración de competencias específicas en estudiantes de psicología.

Teniendo en cuenta que las competencias se integran en los proyectos educativos de cada institución, dándole un matiz particular de acuerdo con su 
misión y visión, en la Corporación Universitaria Minuto de Dios (Uniminuto), las competencias están insertadas en el enfoque praxeológico (Juliao, 2007), que propende por un conocimiento práxico y transformador de la realidad, donde se busque la articulación de saberes y la transdisciplinariedad. En congruencia con este enfoque, la evaluación de las competencias pasa, como lo afirma Tobón (2007), de un énfasis en conocimientos específicos y factuales, a desempeños contextualizados en un determinado entorno.

Se propone entonces el concepto valoración, para resaltar el carácter apreciativo de la evaluación, en un proceso que permite obtener información sobre el grado de adquisición, construcción y desarrollo de las competencias. Esta valoración, más que realizar una medición exacta basada en la aplicación de instrumentos cuantitativos, pretende describir las competencias específicas adquiridas por ellos durante el ciclo de formación profesional, a partir de uno de sus productos académicos: los proyectos e informes finales de práctica.

Las prácticas posibilitan que los estudiantes pongan en contexto los conocimientos construidos durante el proceso de formación y continúen desarrollando las competencias específicas. La práctica profesional en Colombia, está reglamentada por el artículo 67 de la Constitución Política, la Ley 115 de 1994, y las disposiciones reglamentarias y estatutarias respectivas. Según el modelo curricular 3.1 de la Corporación Universitaria Minuto de Dios, se define la práctica profesional, como "el período de la formación de los y las estudiantes, a través del cual tienen la posibilidad de conjugar los conocimientos adquiridos en la academia, con las experiencias propias de los diversos ámbitos de acción de la psicología" (p. 7).
Las prácticas son desarrolladas por los estudiantes durante los cuatro últimos semestres del plan de estudios. Son un momento crucial en la formación profesional, ya que las competencias específicas que fueron desarrolladas previamente en el aula de clase como lugar privilegiado de aprendizaje, se desplazan a un nuevo espacio en que deben confrontarse con las demandas de las distintas instituciones de práctica. Además, es un espacio propicio para valorar la idoneidad profesional según las competencias que el estudiante ha desarrollado para intervenir adecuadamente en diferentes contextos. Por esta razón, una valoración de las competencias en los estudiantes de últimos semestres, que están realizando sus prácticas profesionales, le permite a la institución de educación superior tener una línea de base para tomar decisiones sobre el currículo, los procesos formativos, la capacitación y estrategias docentes, recursos pedagógicos, políticas institucionales, entre otras.

Esta investigación tiene como objetivo valorar las competencias profesionales específicas construidas durante el estudio, en estudiantes de psicología de la Corporación Universitaria Minuto de Dios, sede Bello, que realizaron las prácticas profesionales durante el 2014. En un primer momento se caracterizan las variables referencias bibliográficas, demandas, campos ocupacionales y técnicas, con base en los proyectos de prácticas profesionales e informes finales elaborados por los estudiantes, para construir las competencias profesionales específicas por valorar, a partir de esta información.

\section{Metodología}

Esta investigación tuvo como estrategia metodológica la revisión documental, que toma como fuente primaria de información los documentos, entendidos estos como "cosas que podemos leer y 
que se refieren a algún aspecto del mundo social" (MacDonald \& Tipton, 1993, p. 287). Los materiales documentales para este estudio fueron los proyectos e informes finales de práctica que elaboraron los estudiantes de psicología durante el 2014. Como técnica de análisis de investigación documental se implementó el análisis de contenido que, según Galeano (2007), buscaba construir categorías y contextualizar la información. Al inicio del estudio se utilizaron técnicas cuantitativas para caracterizar las variables, como el uso de estadísticos descriptivos $y$, a partir de ellos, se categorizó la información para construir las competencias para valorar.

El material de análisis consistió en 259 proyectos y 259 informes finales de prácticas. Cada proyecto con su correspondiente informe final se considera como un solo documento. Se tomaron entonces para este estudio 259 unidades documentales. Estos textos corresponden al
$100 \%$ de los documentos entregados a la facultad en el año respectivo. Inicialmente se hizo lectura de 15 proyectos de prácticas y sus correspondientes informes finales, elegidos al azar. A partir de estos se identificaron 4 variables con el fin de sistematizar la información en una matriz. Las variables fueron: referencias bibliográficas, demandas, campos ocupacionales y técnicas.

Se presentan a continuación cada una de las variables con su respectiva clasificación y los datos arrojados.

\section{Referentes}

Se clasificó según el área de conocimiento del texto citado (psicología, educación, otras áreas del conocimiento y sin citas) y tipo de texto citado (teóricocontemporáneo, clásico, resultados de investigación, texto sin acreditación académica).

Tabla 1. Clasificación según referencias bibliográficas

$\begin{array}{cccr}\begin{array}{c}\text { Referencias } \\ \text { bibliográficas }\end{array} & \text { Psicología } & \begin{array}{c}\text { Número de } \\ \text { proyectos }\end{array} & \text { Media } \\ & \text { Otras áreas del conocimiento (economía, gestión } & 156 & 60,2 \% \\ \begin{array}{c}\text { Área del } \\ \text { conocimiento } \\ \text { del texto citado }\end{array} & \begin{array}{c}\text { humana, administración, legislación, ciencias de la } \\ \text { salud, sociología, filosofía, psiquiatría y literatura) }\end{array} & 160 & 61,8 \% \\ & \text { Educación } & 102 & 39,4 \% \\ \text { Tipo de texto } & \text { Sin citas } & 22 & 8,5 \% \\ \text { citado } & \text { Teórico-contemporáneo } & 178 & 68,7 \% \\ & \text { Clásico } & 94 & 36,3 \% \\ & \text { Resultados de investigación } & 12 & 4,6 \%\end{array}$

\section{Técnicas}

La variable "técnicas", en esta investigación, incluye metodologías, estrategias, técnicas, enfoques teóricos y actividades, dado que todas ellas fueron incluidas por los estudiantes en el apartado de técnicas, que se solicita en el formato de prácticas. Por tal motivo, para efectos de este análisis, se sistematizaron todas las mencio- 
nadas dentro de esta variable, tal y como fueron nombradas por los autores de los textos. Los resultados se agruparon en tres tipos de técnicas: psicológicas, investigativas, y "otras técnicas", que incluye técnicas lúdicas, artísticas y otros procedimientos que no son de uso exclusivo de los profesionales en psicología (tabla 2). En las técnicas psicológicas se incluyeron aquellas que fueron desarrolladas por las diferentes escuelas teóricas de la psicología, y en los textos se encontraron el taller reflexivo, el grupo focal, la aplicación de test psicométricos y proyectivos, el grupo operativo, entre otros. En las investigativas se hallaron la observación participante, la entrevista, la revisión documental, entre otras. Como "otras técnicas" se identificaron el cine foro, el sociodrama, los juegos de roles, la pintura, entre otras.

Tabla 2. Tipo de técnica

Tipo de técnica

Psicológicas

Investigativas

Otras técnicas
Número de proyectos que utilizaron técnicas

177

42

216
Media

$68,3 \%$

$16,2 \%$

$83,4 \%$

\section{Campos ocupacionales}

Se identificaron, a partir del tipo de agencia, los campos ocupacionales en los que los estudiantes realizaron sus prácticas profesionales (tabla 3). Se establecieron de esta manera seis campos: educativo, social-comunitario, organizacional, jurídico, clínico e investigación.

Tabla 3. Campos ocupacionales

$\begin{array}{ccc}\text { Campo ocupacional } & \text { Número de proyectos (N) } & \text { Media } \\ \text { Educativo } & 119 & 45,9 \% \\ \text { Social - comunitario } & 48 & 18,5 \% \\ \text { Organizacional } & 41 & 15,8 \% \\ \text { Jurídico } & 23 & 8,9 \% \\ \text { Clínico } & 16 & 6,2 \% \\ \text { Investigación } & 12 & 4,6 \% \\ \text { TOTAL } & 259 & 100 \%\end{array}$

\section{Demandas}

Las demandas que hacen las agencias de prácticas a los estudiantes, se organizaron según el campo ocupacional al que pertenece dicha agencia (tabla 4).
Las demandas hacen referencia a las problemáticas, necesidades o procesos que debe atender el estudiante en prácticas y que tienen relación con la población objeto de intervención. 
Campo ocupacional

Educativo

Organizacional

Jurídico

Clínico

Investigación
Tipo de demanda

Consumo de sustancias psicoactivas, convivencia, formación en valores, habilidades para la vida, intervención familiar, proyecto de vida, rendimiento académico, educación sexual, deserción escolar y promoción, y prevención en salud.

Convivencia, procesos de construcción de identidad y fortalecimiento de procesos comunitarios, proyecto de vida, inclusión social, educación sexual, protección de la infancia, pautas de crianza, consumo de sustancias psicoactivas, intervención familiar y formación en valores.

Capacitación, evaluación del desempeño laboral, prevención de riesgos laborales, inducción laboral, procesos de selección, administración de recursos humanos, procesos de calidad.

Salud mental, reconstrucción del sentido de vida, violencia intrafamiliar, inclusión social, bienestar social y laboral, consumo de sustancias psicoactivas, proyecto de vida, educación sexual, abuso sexual.

Intervención familiar, psicoterapia de apoyo, salud mental, evaluación y diagnóstico, intervención en crisis, aplicación de test, asesoría individual, problemas de aprendizaje.

Formulación de proyectos de investigación, promoción de la investigación, semilleros de investigación, ejecución de proyectos de investigación.
La sistematización de las variables permitió establecer relaciones y construir, a partir de ellas, las competencias por valorar: fundamentación teórica (a partir del área de conocimiento del texto citado), búsqueda bibliográfica (según el tipo de texto citado), recursos de intervención (a partir de las técnicas), y articulación teórico-contextual (a partir de las referencias bibliográficas, las demandas, el campo ocupacional y las técnicas)

\section{Hallazgos}

\section{Fundamentación teórica}

La competencia "fundamentación teórica" se construyó a partir de todos aquellos textos que los estudiantes utilizaron para fundamentar sus intervenciones desde un campo disciplinar particular.
Esta competencia permite que los procesos y productos académicos elaborados por los estudiantes estén estructurados de forma coherente y rigurosa, de manera que sus acciones estén justificadas y organizadas, y que respondan a una cosmovisión particular de los fenómenos que se desarrollan, en este caso, a partir de la formación psicológica. La fundamentación teórica constituye el eje central del saber profesional, ya que a partir de ella se comprenden los fenómenos, se ordenan, y se construyen los distintos instrumentos para su intervención.

La competencia "fundamentación teórica" fue valorada a partir del análisis de las referencias bibliográficas incluidas por los estudiantes en los proyectos de práctica, según las áreas del conocimien- 
to del texto citado. Los referentes teóricos se constituyen como un insumo para estructurar los proyectos de práctica. Esta estructura le da sentido a la lectura de los fenómenos abordados por ellos, y a la planeación de las distintas estrategias que utilizan para intervenirlos.

La fundamentación teórica hace referencia a los recursos teóricos con que cuentan los estudiantes para hacer lectura de las problemáticas sociales. En el caso de los proyectos de práctica, se espera que, al ser una práctica en psicología, los estudiantes fundamenten dichos proyectos, haciendo referencia a textos que teoricen desde este campo disciplinar. Se encontró que, aunque la mayor parte de los proyectos contiene al menos una cita de textos de la psicología $(60,2 \%$ de los documentos), un $39,8 \%$ restante no incluyó ninguna cita que corresponda específicamente a los desarrollos de su propia disciplina. Este hallazgo abre el interrogante sobre la frecuencia con que los estudiantes se remiten a las teorizaciones propias de su disciplina, tanto para realizar lecturas del contexto como para planear y ejecutar sus intervenciones en la práctica psicológica.

Llama la atención que se encontró un alto porcentaje de proyectos de práctica que se remiten a textos de otras áreas del conocimiento, en especial, de la educación $(39,4 \%)$ que es uno de los campos con mayor presencia de estudiantes de prácticas $(45,9 \%)$. Se reconoce entonces un esfuerzo por acercarse al contexto desde una mirada que recoja los aportes teóricos de diversas disciplinas, en especial, las que han teorizado mayoritariamente sobre el campo en que se van a desempeñar.

Se encontró citación de autores provenientes de áreas del conocimiento tan diversas como la economía, gestión humana, administración, ciencias de la salud, sociología, filosofía, psiquiatría y literatura. Esto implica también que hay un esfuerzo de los estudiantes por vincular autores de distintas áreas del conocimiento, promoviendo una lectura de los fenómenos y una intervención de carácter inter-disciplinar. Es importante también el porcentaje, aún significativo, de proyectos sin citas ni referencias bibliográficas $(8,5 \%)$, que pone contundentemente en cuestión la calidad de los proyectos con relación a la necesidad de fundamentarlos teóricamente, pues es sabido que la referenciación bibliográfica permite darle soporte y solidez a cualquier producto académico.

\section{Búsqueda bibliográfica}

La búsqueda bibliográfica se refiere a las distintas estrategias de consulta que utiliza el estudiante para fortalecer la dimensión teórica de sus proyectos de práctica, de manera que su intervención esté basada, no solo en autores o teorías particulares, sino también en investigaciones actuales, contextuales y que permitan enriquecer las intervenciones e incorporar otras nuevas. La búsqueda bibliográfica amplía los horizontes de comprensión que el estudiante hace de los fenómenos que interviene en su práctica.

Se encontró en esta competencia que los estudiantes de práctica citan un alto porcentaje de textos con acreditación académica, incluyendo textos de carácter teórico contemporáneos $(68,7 \%)$, clásicos $(36,3 \%)$ y resultados de investigación $(4,6 \%)$. Los textos sin acreditación académica tienen un bajo porcentaje de citación $(4,6 \%)$, pero son de todas formas utilizados por estudiantes, incluyendo páginas web y blogs con contenidos de carácter informal, planteando interrogantes sobre la rigurosidad de estos proyectos de práctica.

Es necesario subrayar el alto porcentaje de citación de textos contemporáneos con relación al de los clásicos, lo que 
pone en evidencia que, por un lado, los autores clásicos no han perdido vigencia y que, por otro lado, los contemporáneos comienzan a ganar un espacio importante en los productos académicos de los psicólogos en formación. Cabe destacar el bajo porcentaje de proyectos que citan resultados de investigación, lo que da cuenta de la escasa referencia a los avances investigativos que podrían dar luces sobre nuevas comprensiones de los fenómenos, la eficacia de algunas intervenciones y las nuevas propuestas sobre técnicas, herramientas y estrategias de intervención.

\section{Recursos de intervención}

Se comprende como una competencia orientada al uso de técnicas para la intervención psicológica. Se construyó a partir de las técnicas que los estudiantes reportaron utilizar en las prácticas profesionales. Cobra importancia en el período de prácticas profesionales, pues es justo ahí cuando el estudiante hace la transición de la formación teórica a la intervención en los campos ocupacionales. Esta intervención se espera que esté fundamentada en sistemas teóricos que han formulado una amplia variedad de técnicas congruentes con la especificidad de su visión ontológica, epistemológica y metodológica (Guba \&Lincoln, 2000).

En los textos se evidenció que no hay diferenciación entre una técnica de intervención, una estrategia metodológica, una actividad o un enfoque teórico, como es el caso de la asesoría individual, la terapia grupal y las campañas de salud mental, que fueron mencionadas en el apartado de técnicas, pero que no constituyen técnicas propiamente dichas, sino estrategias metodológicas que pueden, a su vez, contener diferentes técnicas que dependerán del enfoque teórico que adopte el profesional a cargo.
Las técnicas psicológicas fueron las que se reportaron con mayor frecuencia en los textos $(68,3 \%)$, sin embargo, el mayor porcentaje de proyectos $(83,4 \%)$ referencian técnicas que no pertenecen al campo disciplinar de la psicología y que corresponden, en su mayoría, a actividades o metodologías de las cuales puede hacer uso otro facilitador grupal sin necesaria formación psicológica. Entre ellas se incluyeron técnicas de carácter lúdico y artístico. Este análisis abre la pregunta sobre la especificidad de la intervención en psicología, su diferencia y la relación con las intervenciones de otros profesionales, ya que muchas de las técnicas citadas por los estudiantes provienen de diferentes campos disciplinares. Cabe resaltar que hay un porcentaje significativo de textos que abordaron técnicas investigativas $(16,2 \%)$, aún en escenarios en los que se desarrollaron prácticas en intervención, como escuelas y organizaciones. De esta manera, técnicas como la entrevista, la encuesta y el grupo focal, fueron puestas al servicio de la intervención, además de ser utilizadas en los espacios destinados exclusivamente a la práctica en investigación (semilleros y grupos de investigación).

\section{Articulación teórico-contextual}

La articulación teórico-contextual hace referencia a la capacidad de integrar los fenómenos sociales con las construcciones teóricas y metodológicas, tanto en su comprensión como en la intervención. Esta competencia fue valorada a partir del análisis de las variables "Área de conocimiento del texto citado", "Tipo de texto", "Demandas" y "Técnicas", lo que permitió reflexionar sobre la suficiencia de los insumos teóricos y metodológicos para hacerle frente a las diversas demandas a las que se enfrenta el estudiante en las agencias de prácticas.

Se encontró amplia diversidad y variabilidad en las demandas que fueron 
formuladas a los estudiantes durante el período de prácticas, a partir de las cuales se les solicitó emprender procesos de intervención. También se evidenció que algunas de ellas son comunes a diferentes campos ocupacionales. En especial, el consumo de sustancias psicoactivas (SPA), la educación sexual y la intervención familiar fueron las temáticas que generaron mayor demanda por parte de las agencias; estas cruzan los campos educativo, social-comunitario, jurídico y clínico. El acompañamiento psicológico a procesos como convivencia, inclusión social, proyecto de vida, formación en valores y salud mental también fue demandado desde diferentes campos ocupacionales.

Así mismo, se identificó que en especial los campos educativo y socialcomunitario comparten la mayoría de demandas (Consumo de SPA, convivencia, formación en valores, intervención familiar, proyecto de vida y educación sexual), abriendo entonces la pregunta sobre la especificidad de la acción psicológica en cada campo ocupacional, y la necesidad de formación diferencial para la intervención en cada campo o, por el contrario, privilegiar una formación psicológica general que responda a las demandas haciendo lectura de contextos. Las demandas más específicas fueron hechas desde los campos organizacional, clínico e investigativo.

Es importante señalar que muchas de estas demandas requieren de conocimientos especializados y niveles de profundización que provean insumos, tanto técnicos como teóricos para la intervención y que no han sido cursados por los estudiantes en pregrado. Sin embargo, la variedad y complejidad de demandas encontradas en este estudio ameritan una reflexión sobre los límites de las solicitudes que pueden hacérsele a un estudiante que ha recibido formación en psicología general, y que aún no cuenta con niveles de especialización, para atender problemáticas que requieren grados más elevados de conocimientos y experiencia en el campo.

Se observa además que las técnicas nombradas por los estudiantes no son suficientes para atender las numerosas demandas que son formuladas por las agencias de prácticas, en vista de que los textos no dan cuenta de la utilización de técnicas que articulen procesos de diagnóstico, intervención y cierre, que permitan responder con rigor técnicocientífico a las necesidades que cada agencia presenta.

Se encontró también una tendencia a abordar las problemáticas desde diversas perspectivas y campos disciplinares, lo que se erige como una fortaleza para la lectura de los fenómenos a la luz de propuestas teóricas diversas y la posibilidad de realizar lecturas interdisciplinares. Así mismo, se vislumbra una brecha entre las referencias teóricas citadas y las técnicas nombradas, lo que da cuenta de algunas limitaciones al momento de traducir las propuestas teóricas en técnicas específicas de intervención.

\section{Discusión}

Durante la investigación se encontró que las demandas hechas a los estudiantes son bastante complejas y por su naturaleza atraviesan distintos campos disciplinares, como es el consumo de sustancias psicoactivas que implica la intervención de profesionales de la psicología, la psiquiatría, la salud ocupacional; además de las temáticas de educación sexual e intervención familiar. En este sentido, la psicología, al igual que los demás saberes, se encuentra en la tarea de transitar de unos conocimientos aislados y fragmentados, a una integración de teorías y perspectivas, que permiten intervenir la variedad de los procesos individuales e institucionales, para evitar, 
como afirma Morin (1999), la simplificación que mutila aquellas realidades que se pretenden comprender e intervenir.

Estos hallazgos nos remiten a una tensión en la formación de los profesionales en psicología, que se debate entre: la formación especializada, que privilegie la profundización en temáticas específicas; o la formación en psicología general, que enfatice el conocimiento de procesos básicos y las lecturas en contexto, más que temáticas particulares.

Se puede conjeturar que la inclusión de diversas áreas del conocimiento o diversos autores en un mismo proyecto e informe de práctica, señala un ejercicio inter-disciplinar hecho por los estudiantes que se espera que no responda exclusivamente a la suma de elementos sino a un enriquecimiento de la intervención en las agencias de práctica. Así, como lo conceptualiza Fernández-Ríos (2010), la apuesta de la formación actual se dirige hacia un trabajo interdisciplinar que consiste en "un proceso teórico-práctico interactivo crítico orientado a la utilización de habilidades cognitivo-emocionales para cambiar perspectivas epistemológicas e integrar y sintetizar conocimiento de diferentes disciplinas para afrontar la complejidad del proceso de solución de los problemas reales" (p. 159).

Frente a la diversidad de demandas encontradas, se considera pertinente emprender investigaciones sobre el tipo de requerimientos que se le hacen al psicólogo de hoy, y la necesidad de formación específica para atender muchos de ellos, cuando los conocimientos en psicología general construidos en el pregrado resultan ser insuficientes por la naturaleza específica de la intervención. Es importante también que las universidades comiencen a revisar y a establecer criterios para delimitar las problemáticas que pueden ser abordadas por los estudiantes en un nivel de formación de prácticas profesionales, teniendo en cuenta que algunas demandas requerirán un nivel de formación posgraduada para poder ser gestionadas de manera apropiada. Esta tarea necesitará de formulación de nuevas regulaciones al ejercicio profesional que contemplenrequerimientos en el nivel de formación para la atención de algunas problemáticas específicas.

Es importante también resaltar la importancia de la formación práctica, y el entrenamiento del estudiante en la implementación de procedimientos que le permitan, además de realizar lecturas teóricas, desarrollar habilidades para la intervención. De esta manera, se limita el uso de estrategias "comodín", que responden más a saberes del sentido común, y que son utilizadas cuando los estudiantes no cuentan con insumos suficientes para enfrentar los retos que se presentan en su práctica profesional. Así mismo, se hace necesario realizar futuras investigaciones orientadas a sistematizar las técnicas de intervención psicológica, de manera que la comunidad académica cuente con documentos compilatorios que permitan condensar el conocimiento que se haya desarrollado sobre la intervención y que recoja técnicas psicológicas apropiadas para los procesos que realizan los profesionales de esta disciplina.

Cabe resaltar el bajo número de citación de investigaciones en los proyectos e informes de práctica, lo cual indica el uso limitado que hacen los estudiantes de las bases de datos para la búsqueda de investigaciones en aras de fundamentar sus intervenciones con criterios técnico-científicos. Este hallazgo abre un interrogante sobre el uso que hacen los profesionales en formación de procedimientos de intervención actualizados que hayan sido objeto de comprobación mediante ensayos clínicos y estudios sobre sus posibles resultados, además de revisar investigaciones que actualicen los desarrollos teóricos de su disciplina 
y propongan nuevos instrumentos de diagnóstico e intervención.

Frente al uso extendido del internet como plataforma privilegiada para la difusión científica y el requerimiento de actualización permanente que el medio le hace al profesional, Ilama la atención que los estudiantes se remitan con poca frecuencia a las novedades investigativas en su campo, considerando que las instituciones de educación superior cuentan con suscripciones que brindan la posibilidad de hacer uso de las bases de datos. En este sentido, Naranjo destaca que "no se ha logrado que los profesores $y$, por ende, los estudiantes universitarios, utilicen los SID [sistemas de información documental] como medios eficaces que posibilitan la estructuración de los datos y la información en conocimiento" (2010, p. 12).

Finalmente, se resalta la necesidad de continuar realizando investigaciones que permitan valorar las competencias profesionales, en especial los insumos teóricos y metodológicos con que los estudiantes cuentan al llegar a un momento crucial en el ciclo de formación universitaria, el periodo de prácticas profesionales, teniendo en cuenta que en esta fase se asiste a nuevos escenarios en el proceso formativo, al ser este el último momento de la formación de pregrado, y que es importante articular estas competencias a los requerimientos del contexto.

\section{Referencias}

Climént, J. B. (2014). Supuestos básicos del enfoque de competencias en educación superior. La educación veterinaria como marco de análisis. Revista Electrónica de Investigación Educativa, 16(1). Recuperado de http://redie.uabc.mx/vol16no1/ contenido-climent14.html

Congreso de La República de Colombia. (1994). Ley 115 de febrero 8 de 1994. Ministerio de Educación Nacional.

Fernández-Ríos, L. (2010). Del conocimiento: ¿Más allá de Bolonia? Innovación Educativa, (20), 157-166.

Galeano, M. E. (2007). Estrategias de intervención: El giro en la mirada. Medellín: La Carreta.

Guba, E., \& Lincoln, Y. (2000). Paradigmas en competencia en la investigación cualitativa. En C. A. Denman \& J. A. Haro (Eds.). Por los rincones: antología de métodos caulitativos en la investigación social. Hermosillo: El Colegio de Sonora.

Gutierrez, G. (2002). El taller reflexivo. Medellín: Universidad Pontificia Bolivariana.

Juliao, C. G. (2007). La praxeología: Una teoría de la práctica. Bogotá: Uniminuto.

MacDonald, K., y Tipton, C. (1993). Using documents. London: Sage.

Morin, E. (1999). El método. El conocimiento del conocimiento. Madrid: Cátedra.

Naranjo, E. (2010). Uso de los sistemas de información documental en la educación superior: estado del arte. Información, Cultura y Sociedad, 22, 11-42. Recuperado de http://www.scielo.org. ar/scielo.php?script $=$ sci arttext\&pid $=$ S 1851 . $17402010000100002 \&$ lng $=\bar{e}$ s\&tlng $=$ es

República de Colombia. (2002). Ley 749. Recuperado de http://www.alcaldiabogota.gov.co/sisjur/normas/ Norma1.jsp?i=6833

Tobón, S. (2006). Aspectos básicos de la formación basada en competencias. (Proyecto Mecesup). Talca.

Tobón, S. (2007). Formación basada en competencias. Pensamiento complejo, diseño curricular y didáctica (2a ed.). Bogotá: Ecoe Ediciones. 